lower part of the ileurn. The intestine below the stricture was very small, flat and empty, while above it was remarkably dilated, looking like a distended colon, its wall, from hypertrophy, being as thick as that of the stomach. A lateral anastomosis was made between the loop above and the one below the stricture, about $30 \mathrm{~cm}$. being excluded from the intestinal current. Senn's large size decalcified bone plates were used in the operation. The recovery from the operation was rapid and without incident. $\mathrm{He}$ regained his health at once and made the most remarkable gain in weight, from 85 to 190 pounds in eleven months.

$\mathrm{He}$ continued in good health for about four years, when the effects of chronic renal and cardiac disease, aggravated or perhaps superinduced by his habits, began to show themselves, and from this time on the usual course of these diseases was passed throughgeneral anasarca, ascites with repeated tappings, etc., and finally death in December, $1896^{\circ}$, exactly five years after the operation. At no time was there any trouble with his intestinal tract after the operation. The specimen still shows the thick cicatricial mass forming the stricture and allowing but a small probe to pass.

The loop of intestine which was excluded has shrunken or atrophied to but a few $\mathrm{cm}$. in length. The anastomotic opening is of good size, having contracted but little, and permits a free unobstructed passage of the intestinal contents. The great dilatation of the bowel above the stricture has all disappeared, the gut being now of uniform size. This is, so far as I am aware, the oldest specimen - five years - of a lateral anastomosis on record.

The cause of the stricture was in all probability cicatricial contraction following an intestinal ulcer, but the history of the patient gave no clue to the primary trouble, and no examination of the body or intestines was permitted after death.

\section{A CASE OF DEFECT IN THE VENTRICULAR SEPTUM AND STENOSIS OF THE PUL- MONARY CONUS IN A MAN THIRTY. TWO YEARS OLD, WITH DEMONSTRATION OF THE HEART.}

Read at the Meeting of the Chicago Pathological Society, April 12, 1897. BY JOSEPHINE E. YOUNG, M.D. CHICAGO, ILI.

October 2, 1896, a patient, 32 years of age, entered Cook County hospital, Chicago, with evidences of an acute, febrile disease. $\mathrm{He}$ gave the following history: When 2 years old a consultation of physicians was held-the child always having been so delicate that it was necessary to carry him about on a pillow, at that time being very poorly nourished and developed, unable to walk, and having at frequent intervals attacks of marked cyanosis. The consultants told his mother that he could not live to maturity. At the age of 17, Prof. Lyman of Rush Medical College demonstrated the case in his clinic as one of cardiac disease. During his entire life, upon marked exertion or excitement, the patient had attacks of pronounced cyanosis.

Upon entrance to the hospital, physical examination revealed the following: General development and nourishment good, clubbed finger ends, color ruddy, no evidence of cyanosis at this time. The pulse was full and regular, later becoming irregular and distinctly subingual. Heart: percussion, relative dulness right border to the right of the right sternal line, base third rib, left border left mammillary line. There was increased dulness when the patient leaned forward. One month later the dulness extended two $\mathrm{cm}$. to the left of the left mammillary line. Palpation: upon entrance there was distinct fremitus over the entire precordia. The apex beat was strong and regular. One month later there was marked arrhythmia. Auscultation: a very loud systolic murmur could be heard over the entire thorax, anteriorly and posteriorly, the maximum intensity being at the junction of the third rib with the left border of the sternum. The murmur was heard very distinctly along the entire left. sternal border. There was no accentuation of the second pulmonic tone. Notwithstanding the fact that for eighteen days the temperature remained at or near 103 ; there was never any cyanosis. The urine was negative, both as to albumin and morphologic elements. Other findings bear no especial relation to the cardiac lesions, the diagnosis of the acute disease halting between acute endocarditis and typhoid fever. Dr. James B. Herrick at this time made a diagnosis of congenital heart disease.

The patient was discharged Nov. 24, 1896, after recovery from the acute attack. Dec. 12, 1896, be reëntered the hospital with facial erysipelas.

The heart and lung findings were as before. In a few days, with a temperature of 104 degrees, cyanosis became a marked feature. The heart was very arrhythmic, the pulse correspondingly irregular and compressible. The spleen extended five $\mathrm{cm}$. below the left costal arch, the liver one and one-half $\mathrm{cm}$. below the right costal arch and in the mammillary line. Examination of the urine showed albumin in rather large amount and granular casts. The extremities were not edematous. Two days before death the dyspnea was extreme and respirations increased to fifty-two. The patient's critical condition and constant right decubitus prevented a thorough examination of the right lung, which later showed evidences of pneumonia. He was rational throughout.

Death occurred Jan. 12, 1897, after one: month's illness, during which time the temperature was frequently 104 and 105 degrees.

The clinical diagnosis was facial erysipelas, acute splenitis, nephritis, pneumonia (?), general septic infection, and congenital heart disease. The autopsy was mrde by Dr. Ludwig Hektoen, Jan. 18, 1897.

Anatomic diagnosis: healed facial erysipelas, lobar pneumonia, embolic abscess in the left lung, acute splenic tumor, chronic and acute nephritis, chronic gastritis, pigmented Peyer's patches, hypertrophy of the heart, chronic and acute endocarditis of the tricuspid and pulmonary valves and the wall of the right ventricle, stenosis of the pulmonary conus and congenital defect of the ventricular septum.

The bacteriologic examination of the organs showed staphylococci pyogenes albus and citreus to be present everywhere.

The cardiac findings more particularly are the following: The hypertrophy is rather marked; the heart weighs 400 grams; the apex is composed equally of the right and left ventricles; the wall of the right auricle measures $4 \mathrm{~mm}$. in the thickest part, that of the left auricle $3 \mathrm{~mm}$; the wall of the right ventricle averages $1 \mathrm{~cm}$. in thickness, that of the left ven- 
tricle $1 \frac{1}{3} \mathrm{~cm}$; the circumference of the aorta at the valvus is $5.7 \mathrm{~cm}$., that of the pulmonary artery is 5.4 c.m.; the ductus Botalli is closed; the arrangement of the pulmonary artery is normal, that of the aorta, which communicates only with the left ventricle, is normal also. Four cm. below the upper margin of the pulmonic semi-lunar valves there is a stenosis of the pulmonary conus, due to fibrous tissue. The lumen of the conus measures $1 \mathrm{~cm}$. in its largest diameter; the foramen ovale is patent, admitting a lead pencil through its orifice; a sickle-shaped valve covers it. Directly below the semi-lunar valves of the aorta there is a defect in the ventricular septum which measures $2.6 \mathrm{~cm}$. in the horizontal dlameter, $2 \mathrm{~cm}$. in the vertical diameter. This defect corresponds to the septum membranacum, or undefended space, the last part of the ventricular septum to close, composed normally of a thin layer of fibrous tissue covered with endothelium. The pulmonary valves are thickened; the free margins of the posterior segment are covered by large polypoid vegetations. These spring also from the ventricular surfaces of the valves and from the wall of the pulmonary artery. The free margins of the tricuspid valves are thickened. Here then are distinct polypoid vegetations. The orifice admits four finger-tips easily; the right auricular appendix shows some irregular masses; the aorta valves are free from thickening; the aorta shows no atheroma; the mitral valve is normal, admitting four finger-tips snugly; the myocardium, except at the stenosis of the conus, is normal. Microscopically, the striæ and nuclei of the fibers are normal, but there is a general segmentation.

The clinical diagnosis of congenital heart disease was based upon the history, the cyanosis, hypertrophy of the right heart, position of the systolic murmur and thrill at the junction of the left third rib with the sternum, the intensity and widespread transmission of the thrill and the weak second pulmonic tone.

As to the formation of the lesions, Fraentzel, Bamberger and Oppolzer support the Hunter-Morgagni theory, a myo- or endo-carditis occurring before the eighth week of fetal life is the cause of the stenosis of the pulmonary artery or its conus. This greatly increases blood pressure by preventing the proper escape of the blood from the right ventricle, the blood necessarily forcing its way into the left ventricle through the undeveloped septum. If the opening in the septum is sufficiently large blood pressure in the right heart is compensated for-there may be no opening (in the septum) of the foramen ovale. If not large enough there will be hypertrophy and dilatation of the right ventricle, stasis in the right auricle, with passage of blood through the foramen ovale, which remains patent.

The case is of interest not so much from its rarity as from the following points: The patient had reached the age of 32 , was a strong, well-developed man doing the ordinarily heavy work of a laborer, had passed through an acute febrile attack of eighteen days' duration without any evidence of cyanosis, had resisted for a month profound erysipelatous and septic infection and had entirely escaped pulmonary tuberculosis, a condition frequent in these cases, owing to the diminished supply of blood sent to the lungs.

A Progressive Potentate.-The young Emperor of China, with a lost faith in the traditional remedies of his people, has dispatched to the medical centers of Europe a large number of students. London, Paris, Berlin and Vienna have already received a respectable installment.

\section{ADAPTATION AND INSERTION OF AN ARTIFICIAL EYE; ENUCLEATION, SYMPATHETIC PHENOM-} ENA, ETC.

BY WILLIAM B. MEANY, M.D.

MEMBER OF: THE AMERICAN MEDICAL ASSOCIATION, ETC. BAINT LOUIS, Mo.

The exercise of a little care with "troublesome" cases in the taking of a caste-either with dentist wax or with superfine dental plaster-of-paris-of the tissue walls of the orbital cavity resulting from the removal of an eyeball, and the artificial eye to be inserted fashioned on the lines of the "model," may overcome the difficulties complained of by advocates of dangerous substitute proceedings for the benign operation of enucleation.

An artificial eye should be fabricated to meet the requirements of the anatomic conformation of the orbital cavity, rather than the adaptation of the tissue walls to a misfit or bespoke eye-which has never been known to vary from its present shape for half a century or thereabouts.

By the adoption of this plan of procedure hazardous and unwarrantable "operations" that permit the retention of a portion of the structure of an eyeball that has becorne or is liable to take on disease at any moment, and thereby incite sympathetic phenomena, will be avoided; and the present unsatisfactory process of "grinding down" of the shop (stock) eye be dispensed with.

The insertion of an artificial eye is very simple and is soon learned by the patient. It must first be steadily pushed beneath the upper eyelid and held there while the lower lid is brought around its lower edges.

Its removal is readily facilitated by depressing the lower lid so as to expose the lower edge of the artificial eye, when it usually slips out by its own weight, and should be caught in a handkerchief or permitted to drop out upon a bed. An artificial eye should not be adapted or inserted until the cicatrix of the conjunctiva and other tissues of the orbit become firm, quiet and free from ulceration or inflammatory discharges. By selecting an artificial eye somewhat smaller than the remaining or sound eye, the distorted or over-exaggerated appearance is avoided. The eye should not be worn continuously-that is, never during bedtime.

This operation or excision of the eyeball is usually performed as follows: A fold of the conjunctiva on the inner or nasal side of the eye having been seized with the forceps is divided with a scissors at the corneo-scleral juncture along its entire circumference. The tendons of the muscles caught up with a strabis. mus hook and divided, the globe turned firmly outward and forward; the scissors (those curved on the flat being used), introduced either on the nasal or temporal side of the eye as the operator may elect, the blades of the scissor being separated so as to straddle the optic nerve, permitting of easy division. Care should be taken to excise the nerve some distance from its ocular attachment.

The globe being brought out of the orbit, the oblique nerves and vessels are divided. The cavity is then carefully syringed with a cold boric acid or a 1 to 10,000 sublimate lotion, or both; the slight hemorrhage that follows the operation is usually arrested by the "syringing" of the cold antiseptic solutions. 